\title{
Weak Embeddable Hypernear-Rings
}

\author{
Jelena Dakić ${ }^{1}$, Sanja Jančić-Rašović ${ }^{1} \mathbb{D}$ and Irina Cristea ${ }^{2, * \mathbb{D}}$ \\ 1 Department of Mathematics, Faculty of Natural Science and Mathematics, University of Montenegro, \\ 81000 Podgorica, Montenegro \\ 2 Centre for Information Technologies and Applied Mathematics, University of Nova Gorica, \\ 5000 Nova Gorica, Slovenia \\ * Correspondence: irina.cristea@ung.si or irinacri@yahoo.co.uk; Tel.: +386-0533-15-395
}

Received: 28 June 2019; Accepted: 18 July 2019; Published: 1 August 2019

\begin{abstract}
In this paper we extend one of the main problems of near-rings to the framework of algebraic hypercompositional structures. This problem states that every near-ring is isomorphic with a near-ring of the transformations of a group. First we endow the set of all multitransformations of a hypergroup (not necessarily abelian) with a general hypernear-ring structure, called the multitransformation general hypernear-ring associated with a hypergroup. Then we show that any hypernear-ring can be weakly embedded into a multitransformation general hypernear-ring, generalizing the similar classical theorem on near-rings. Several properties of hypernear-rings related with this property are discussed and illustrated also by examples.
\end{abstract}

Keywords: hypernear-ring; multitransformation; embedding

\section{Introduction}

Generally speaking, the embedding of an algebraic structure into another one requires the existence of an injective map between the two algebraic objects, that also preserves the structure, i.e., a monomorphism. The most natural, canonical and well-known embeddings are those of numbers: the natural numbers into integers, the integers into the rational numbers, the rational numbers into the real numbers and the real numbers into the complex numbers. One important type of rings is that one of the endomorphisms of an abelian group under function pointwise addition and composition of functions. It is well known that every ring is isomorphic with a subring of such a ring of endomorphisms. But this result holds only in the commutative case, since the set of the endomorphisms of a non-abelian group is no longer closed under addition. This aspect motivates the interest in studying near-rings, that appear to have applications also in characterizing transformations of a group. More exactly, the set of all transformations of a group $G$, i.e., $T(G)=\{f: G \rightarrow G\}$ can be endowed with a near-ring structure under pointwise addition and composition of mappings, such a near-ring being called the transformation near-ring of the group $G$.

In 1959 Berman and Silverman [1] claimed that every near-ring is isomorphic with a near-ring of transformations. At that time only some hints were presented, while a direct and clear proof of this result appeared in Malone and Heatherly [2] almost ten years later. Since $T(G)$ has an identity, it immediately follows that any near-ring can be embedded in a near-ring with identity. Moreover, in the same paper [2], it was proved that a group $(H,+)$ can be embedded in a group $(G,+)$ if and only if the near-ring $T_{0}(H)$, consisting of all transformations of $H$ which multiplicatively commute with the zero transformation, can be embedded into the similar near-ring $T_{0}(G)$ on $G$ under a kernel-preserving monomorphism of near-rings.

Similarly to near-rings, but in the framework of algebraic hyperstructures, Dašić [3] defined the hypernear-rings as hyperstructures with the additive part being a quasicanonical hypergroup $[4,5]$ 
(called also a polygroup [6,7]), and the multiplicative part being a semigroup with a bilaterally absorbing element, such that the multiplication is distributive with respect to the hyperaddition on the left-hand side. Later on, this algebraic hyperstructure was called a strongly distributive hypernear-ring, or a zero-symmetric hypernear-ring, while in a hypernear-ring the distributivity property was replaced by the "inclusive distributivity" from the left (or right) side. Moreover, when the additive part is a hypergroup and all the other properties related to the multiplication are conserved, we talk about a general hypernear-ring [8]. The distributivity property is important also in other types of hyperstructures, see e.g., [9]. A detailed discussion about the terminology related to hypernear-rings is included in [10]. In the same paper, the authors defined on the set of all transformations of a quasicanonical hypergroup that preserves the zero element a hyperaddition and a multiplication (as the composition of functions) in such a way to obtain a hypernear-ring. More general, the set of all transformations of a hypergroup (not necessarily commutative) together with the same hyperaddition and multiplication is a strongly distributive hypernear-ring [3]. In this note we will extend the study to the set of all multimappings (or multitransformations) of a (non-abelian) hypergroup, defining first a structure of (left) general hypernear-ring, called the multitransformation general hypernear-ring associated with a hypergroup. Then we will show that any hypernear-ring can be weakly embedded into a multitransformation general hypernear-ring, generalizing the similar classical theorem on near-rings [2]. Besides, under same conditions, any additive hypernear-ring is weakly embeddable into the additive hypernear-ring of the transformations of a hypergroup with identity element that commute multiplicatively with the zero-function. The paper ends with some conclusive ideas and suggestions of future works on this topic.

\section{Preliminaries}

We start with some basic definitions and results in the framework of hypernear-rings and near-rings of group mappings. For further properties of these concepts we refer the reader to the papers $[2,3,11,12]$ and the fundamental books [13-15]. For the consistence of our study, regarding hypernear-rings we keep the terminology established and explained in $[8,16]$.

First we recall the definition introduced by Dašić in 1978.

Definition 1. [12] A hypernear-ring is an algebraic system $(R,+, \cdot)$, where $R$ is a non-empty set endowed with a hyperoperation $+: R \times R \rightarrow P^{*}(R)$ and an operation $:: R \times R \rightarrow R$, satisfying the following three axioms:

1. $(R,+)$ is a quasicanonical hypergroup (named also polygroup [6]), meaning that:

(a) $x+(y+z)=(x+y)+z$ for any $x, y, z \in R$,

(b) there exists $0 \in R$ such that, for any $x \in R, x+0=0+x=\{x\}$,

(c) for any $x \in R$ there exists a unique element $-x \in R$, such that $0 \in x+(-x) \cap(-x)+x$,

(d) for any $x, y, z \in R, z \in x+y$ implies that $x \in z+(-y), y \in(-x)+z$.

2. $(R, \cdot)$ is a semigroup endowed with a two-sided absorbing element 0 , i.e., for any $x \in R, x \cdot 0=0 \cdot x=0$.

3. The operation "." is distributive with respect to the hyperoperation " + " from the left-hand side: for any $x, y, z \in R$, there is $x \cdot(y+z)=x \cdot y+x \cdot z$.

This kind of hypernear-ring was called by Gontineac [11] a zero-symmetric hypernear-ring. In our previous works [10,16], regarding the distributivity, we kept the Vougiouklis' terminology [17], and therefore, we say that a hypernear-ring is a hyperstructure $(R,+, \cdot)$ satisfying the above mentioned axioms 1. and 2., and the new one:

$3^{\prime}$. The operation "." is inclusively distributive with respect to the hyperoperation " + " from the left-hand side: for any $x, y, z \in R, x \cdot(y+z) \subseteq x \cdot y+x \cdot z$. Accordingly, the Dašić 's hypernear-ring (satisfying the axioms 1., 2., and 3.) is called strongly distributive hypernear-ring.

Furthermore, if the additive part is a hypergroup (and not a polygroup), then we talk about a more general type of hypernear-rings. 
Definition 2. [8] A general (left) hypernear-ring is an algebraic structure $(R,+, \cdot)$ such that $(R,+)$ is a hypergroup, $(R, \cdot)$ is a semihypergroup and the hyperoperation "." is inclusively distributive with respect to the hyperoperation " + " from the left-hand side, i.e., $x \cdot(y+z) \subseteq x \cdot y+x \cdot z$, for any $x, y, z \in R$. If in the third condition the equality is valid, then the structure $(R,+, \cdot)$ is called strongly distributive general (left) hypernear-ring. Besides, if the multiplicative part $(R, \cdot)$ is only a semigroup (instead of a semihypergroup), we get the notion of general (left) additive hypernear-ring.

Definition 3. Let $\left(R_{1},+, \cdot\right)$ and $\left(R_{2},+, \cdot\right)$ be two general hypernear-rings. A map $\rho: R_{1} \rightarrow R_{2}$ is called an inclusion homomorphism if the following conditions are satisfied:

1. $\rho(x+y) \subseteq \rho(x)+\rho(y)$

2. $\rho(x \cdot y) \subseteq \rho(x) \cdot \rho(y)$ for all $x, y \in R_{1}$.

A map $\rho$ is called a good (strong) homomorphism if in the conditions 1. and 2. the equality is valid.

In the second part of this section we will briefly recall the fundamentals on near-rings of group mappings. A left near-ring $(N,+, \cdot)$ is a non-empty set endowed with two binary operations, the addition + and the multiplication $\cdot$, such that $(N,+)$ is a group (not necessarily abelian) with the neutral element $0,(N, \cdot)$ is a semigroup, and the multiplication is distributive with respect to the addition from the left-hand side. Similarly, we have a right near-ring. Several examples of near-rings are obtained on the set of "non-linear" mappings and here we will see two of them.

Let $(G,+)$ be a group (not necessarily commutative) and let $T(G)$ be the set of all functions from $G$ to $G$. On $T(G)$ define two binary operations: "+" is the pointwise addition of functions, while the multiplication "." is the composition of functions. Then $(T(G),+, \cdot)$ is a (left) near-ring, called the transformation near-ring on the group $G$. Moreover, let $T_{0}(G)$ be the subnear-ring of $T(G)$ consisting of the functions of $T(G)$ that commute multiplicatively with the zero function, i.e., $T_{0}(G)=\{f \in T(G) \mid$ $f(0)=0\}$. These two near-rings, $T(G)$ and $T_{0}(G)$, have a fundamental role in embeddings. Already in 1959, it was claimed by Berman and Silverman [1] that every near-ring is isomorphic with a near-ring of transformations. One year later the proof was given by the same authors, but using an elaborate terminology and methodology. Here below we recall this result together with other related properties, as presented by Malone and Heatherly [2].

Theorem 1. [2] Let $(R,+, \cdot)$ be a near-ring. If $(G,+)$ is any group containing $(R,+)$ as a proper subgroup, then $(R,+, \cdot)$ can be embedded in the transformation near-ring $T(G)$.

Corollary 1. [2] Every near-ring can be embedded in a near-ring with identity.

Theorem 2. [2] A group $(H,+)$ can be embedded in a group $(G,+)$ if and only if $T_{0}(H)$ can be embedded in $T_{0}(G)$ by a near-ring monomorphism which is kernel-preserving.

Theorem 3. [2] A group $(H,+)$ can be embedded in a group $(G,+)$ if and only if the near-ring $T(H)$ can be embedded in the near-ring $T(G)$.

\section{Weak Embeddable Hypernear-Rings}

In this section we aim to extend the results related to embeddings of near-rings to the case of hypernear-rings. In this respect, instead of a group $(G,+)$ we will consider a hypergroup $(H,+)$ and then the set of all multimappings on $H$, which we endow with a structure of general hypernear-ring.

Theorem 4. Let $(H,+)$ be a hypergroup (not necessarily abelian) and $T^{*}(H)=\left\{h: H \rightarrow P^{*}(H)\right\}$ the set of all multimappings of the hypergroup $(H,+)$. Define, for all $(f, g) \in T^{*}(H) \times T^{*}(H)$, the following hyperoperations:

$$
f \oplus g=\left\{h \in T^{*}(H) \mid(\forall x \in H) h(x) \subseteq f(x)+g(x)\right\}
$$




$$
f \odot g=\left\{h \in T^{*}(H) \mid(\forall x \in H) h(x) \subseteq g(f(x))=\bigcup_{u \in f(x)} g(u)\right\} .
$$

The structure $\left(T^{*}(H), \oplus, \odot\right)$ is a (left) general hypernear-ring.

Proof. For any $f, g \in T^{*}(H)$ it holds: $f \oplus g \neq \varnothing$. Indeed, for any $x \in H$, it holds $f(x) \neq \varnothing$ and $g(x) \neq \varnothing$ and thus, $f(x)+g(x) \neq \varnothing$. Therefore, for the map $h: H \rightarrow P^{*}(H)$ defined by: $h(x)=f(x)+g(x)$ for all $x \in H$, it holds $h \in f \oplus g$. Now, we prove that the hyperoperation $\oplus$ is associative. Let $f, g, h \in T^{*}(H)$ and set

$$
\begin{aligned}
& L=(f \oplus g) \oplus h=\bigcup\left\{h^{\prime} \oplus h \mid h^{\prime} \in f \oplus g\right\}= \\
& =\bigcup\left\{h^{\prime} \oplus h \mid(\forall x \in H) h^{\prime}(x) \subseteq f(x)+g(x)\right\} .
\end{aligned}
$$

Thus, if $h^{\prime \prime} \in L$, then, for all $x \in H$, it holds: $h^{\prime \prime}(x) \subseteq h^{\prime}(x)+h(x) \subseteq(f(x)+g(x))+h(x)$. Conversely, if $h^{\prime \prime}$ is an element of $T^{*}(H)$ such that: $h^{\prime \prime}(x) \subseteq(f(x)+g(x))+h(x)$, for all $x \in H$, and if we choose $h^{\prime}$ such that $h^{\prime}(x)=f(x)+g(x)$ for all $x \in H$, then $h^{\prime} \in f \oplus g$ and $h^{\prime \prime} \in h^{\prime} \oplus h$ i.e., $h^{\prime \prime} \in L$. So, $L=\left\{h^{\prime \prime} \in T^{*}(H) \mid(\forall x \in H) h^{\prime \prime}(x) \subseteq(f(x)+g(x))+h(x)\right\}$. On the other side, take $D=f \oplus(g \oplus h)$. Then, $D=\left\{h^{\prime \prime} \in T^{*}(H) \mid(\forall x \in H) h^{\prime \prime}(x) \subseteq f(x)+(g(x)+h(x))\right\}$. By the associativity of the hyperoperation + we obtain that $L=D$, meaning that the hyperoperation $\oplus$ is associative.

Let $f, g \in T^{*}(H)$. We prove that the equation $f \in g \oplus a$ has a solution $a \in T^{*}(H)$. If we set $a(x)=H$, for all $x \in H$, then $a \in T^{*}(H)$ and for all $x \in H$ it holds $g(x)+a(x)=H \supseteq f(x)$. So, $f \in g \oplus a$. Similarly, the equation $f \in a \oplus g$ has a solution in $T^{*}(H)$. Thus, $\left(T^{*}(H), \oplus\right)$ is a hypergroup.

Now, we show that $\left(T^{*}(H), \odot\right)$ is a semihypergroup. Let $f, g \in T^{*}(H)$. For all $x \in H$ it holds $g(x) \neq \varnothing$ and so $g(f(x)) \neq \varnothing$. Let $h: H \rightarrow P^{*}(H)$ be a multimapping defined by $h(x)=g(f(x))$, for all $x \in H$. Obviously, $h \in f \odot g$ and so $f \odot g \neq \varnothing$. Let us prove that $\odot$ is a associative. Let $f, g, h \in T^{*}(H)$. Set:

$$
\begin{gathered}
L=(f \odot g) \odot h=\bigcup\left\{h^{\prime} \odot h \mid h^{\prime} \in f \odot g\right\}=\left\{h^{\prime} \odot h \mid(\forall x \in H) h^{\prime}(x) \subseteq g(f(x))\right\}= \\
=\left\{h^{\prime \prime} \mid(\forall x \in H) h^{\prime \prime}(x) \subseteq h\left(h^{\prime}(x)\right) \wedge h^{\prime}(x) \subseteq g(f(x))\right\} .
\end{gathered}
$$

So, if $h^{\prime \prime} \in L$, then $h^{\prime \prime}(x) \subseteq h(g(f(x)))$, for all $x \in H$. On the other side, if $h^{\prime \prime} \in T^{*}(H)$ and $h^{\prime \prime}(x) \subseteq$ $h(g(f(x)))$ for all $x \in H$, then we choose $h^{\prime} \in T^{*}(H)$ such that $h^{\prime}(x)=g(f(x))$ and consequently we obtain that $h^{\prime \prime} \subseteq h\left(h^{\prime}(x)\right)$. Thus, $h^{\prime \prime} \in L$. So, $L=\left\{h^{\prime \prime} \in T^{*}(H) \mid(\forall x \in H) h^{\prime \prime}(x) \subseteq h(g(f(x)))\right\}$.

Similarly, $D=f \odot(g \odot h)=\left\{h^{\prime \prime} \mid(\forall x \in H) h^{\prime \prime}(x) \subseteq h(g(f(x)))\right\}$. Thus, $L=D$.

It remains to prove that the hyperoperation $\oplus$ is inclusively distributive with respect to the hyperoperation $\odot$ on the left-hand side. Let $f, g, h \in T^{*}(H)$. Set $L=f \odot(g \oplus h)=\bigcup\left\{f \odot h^{\prime} \mid h^{\prime} \in\right.$ $g \oplus h\}=\bigcup\left\{f \odot h^{\prime} \mid h^{\prime} \in T^{*}(H) \wedge(\forall x) h^{\prime}(x) \subseteq g(x)+h(x)\right\}$. So, if $k \in L$ then for all $x \in H$ it holds: $k(x) \subseteq h^{\prime}(f(x)) \subseteq g(f(x))+h(f(x))$.

On the other hand, $D=(f \odot g) \oplus(f \odot h)=\bigcup\left\{k_{1} \oplus k_{2} \mid k_{1} \in f \odot g, k_{2} \in f \odot h\right\}$. Let $k \in L$. Choose, $k_{1}, k_{2} \in T^{*}(H)$ such that $k_{1}(x)=g(f(x))$ and $k_{2}(x)=h(f(x))$ for all $x \in H$. Then $k_{1} \in f \odot g$ and $k_{2} \in f \odot h$. Thus, $k(x) \subseteq k_{1}(x)+k_{2}(x)$ for all $x \in H$, i.e., $k \in k_{1} \oplus k_{2}$ and $k_{1} \in f \odot g, k_{2} \in f \odot h$. So, $k \in D$. Therefore, $L \subseteq D$.

Definition 4. $T^{*}(H)$ is called the multitransformations general hypernear-ring on the hypergroup $H$.

Remark 1. Let $(G,+)$ be a group and $T(G)$ be the transformations near-ring on $G$. Obviously, $T(G) \subset$ $T^{*}(G)=\left\{f: G \rightarrow P^{*}(G)\right\}$ and, for all $f, g \in T(G)$, it holds: $f \oplus g=f+g, f \odot g=f \cdot g$, meaning that the hyperoperations defined in Theorem 4 are the same as the operations in Theorem 1. It follows that $T(G)$ is a sub(hyper)near-ring of $\left(T^{*}(G), \oplus, \odot\right)$. 
Definition 5. We say that the hypernear-ring $\left(R_{1},+, \cdot\right)$ is weak embeddable (by short $W-$ embeddable) in the hypernear-ring $\left(R_{2},+, \cdot\right)$ if there exists an injective inclusion homomorphism $\mu: R_{1} \rightarrow R_{2}$.

The next theorem is a generalization of Theorem 1 [5].

Theorem 5. For every general hypernear-ring $(R,+, \cdot)$ there exists a hypergroup $(H,+)$ such that $R$ is $W-$ embeddable in the associated hypernear-ring $T^{*}(H)$.

Proof. Let $(R,+, \cdot)$ be a hypernear-ring and let $(H,+)$ be a hypergroup such that $(R,+)$ is a proper subhypergroup of $(H,+)$. For a fixed element $r \in R$ we define a multimapping $f_{r}: H \rightarrow P^{*}(H)$ as follows

$$
f_{r}(g)= \begin{cases}g \cdot r, & \text { if } g \in R \\ r, & \text { if } g \in H \backslash R .\end{cases}
$$

Let us define now the mapping $\mu: R \rightarrow T^{*}(H)$ as $\mu(r)=f_{r}$, which is an inclusion homomorphism. Indeed, if $a, b \in R$ then we have $\mu(a+b)=\left\{f_{c} \mid c \in a+b\right\}$ and $\mu(a) \oplus \mu(b)=f_{a} \oplus f_{b}=\{h \mid(\forall g \in$ H) $\left.h(g) \subseteq f_{a}(g)+f_{b}(g)\right\}$.

Consider $c \in a+b$ and $g \in H$. If $g \in R$, then $f_{c}(g)=g \cdot c \subseteq g \cdot(a+b) \subseteq g \cdot a+g \cdot b=$ $f_{a}(g)+f_{b}(g)$. If $g \in H \backslash R$, then $f_{c}(g)=c \in a+b=f_{a}(g)+f_{b}(g)$. It follows that, for all $g \in H$, we have $f_{c}(g) \subseteq f_{a}(g)+f_{b}(g)$ and therefore $f_{c} \in \mu(a) \oplus \mu(b)$, meaning that $\mu(a+b) \subseteq \mu(a) \oplus \mu(b)$.

Similarly, there is $\mu(a \cdot b)=\left\{f_{c} \mid c \in a \cdot b\right\}$ and $\mu(a) \odot \mu(b)=f_{a} \odot f_{b}=\left\{h \in T^{*}(H) \mid(\forall g \in\right.$ H) $\left.h(g) \subseteq f_{b}\left(f_{a}(g)\right)\right\}$. Let $c \in a \cdot b$. Then, for $g \in R$, it holds: $f_{c}(g)=g \cdot c \subseteq g \cdot(a \cdot b)=(g \cdot a) \cdot b=$ $f_{b}\left(f_{a}(g)\right)$. If $g \in H \backslash R$, then there is $f_{c}(g)=c \in a \cdot b=f_{b}(a)=f_{b}\left(f_{a}(g)\right)$. Thus, $f_{c} \in \mu(a) \odot \mu(b)$ and so $\mu(a \odot b) \subseteq \mu(a) \odot \mu(b)$.

Based on Definition 3, we conclude that $\mu$ is an inclusive homomorphism. It remains to show that $\mu$ is injective. If $\mu(a)=\mu(b)$, then for all $g \in H$, it holds $f_{a}(g)=f_{b}(g)$. So, if we choose $g \in H \backslash R$, then we get that $a=f_{a}(g)=f_{b}(g)=b$.

These all show that the general hypernear-ring $R$ is $W$-embeddable in $T^{*}(H)$.

Remark 2. If $(R,+, \cdot)$ is a near-ring such that $(R,+)$ is a proper subgroup of a group $(G,+)$, then for a fixed $r \in R$ the multimapping $f_{r}$ constructed in the proof of Theorem 5 is in fact a map from $G$ to $G$, since in this case the multiplication - is an ordinary operation, i.e., $g \cdot r \in G$, for all $g \in R$. Thus $f_{r}: G \rightarrow G$ and thereby $\mu(R) \subseteq T(G)$. By consequence $\mu: R \rightarrow T(G)$ is an ordinary monomorphism. In other words, Theorem 5 is a generalization of Theorem 1.

Example 1. Let $(R,+, \cdot)$ be a left near-ring. Let $P_{1}$ and $P_{2}$ be non-empty subsets of $R$ such that $R \cdot P_{1} \subseteq P_{1}$ and $P_{1} \subseteq Z(R)$, where $Z(R)$ is the center of $R$, i.e., $Z(R)=\{x \in R \mid(\forall y \in R) x+y=y+x\}$. For any $(x, y) \in R^{2}$ define:

$$
x \oplus_{P_{1}} y=x+y+P_{1}, \quad x \odot_{P_{2}} y=x P_{2} y .
$$

Then the structure $\left(R, \oplus_{P_{1}}, \odot_{P_{2}}\right)$ is a general left hypernear-ring $[8,18]$. Let $H=R \cup\{a\}$ and define on $H$ the hyperoperation $\oplus_{P}^{\prime}$ as follows:

$$
x \oplus_{P_{1}}^{\prime} y= \begin{cases}x \oplus_{P_{1}} y, & \text { if } x, y \in R \\ H, & \text { if } x=a \vee y=a .\end{cases}
$$

It is clear that $H$ is a hypergroup such that $(R,+)$ is a proper subhypergroup of $(H,+)$. Besides, based on Theorem 5, for every $r \in R$ the multimapping $f_{r}: H \rightarrow P^{*}(H)$ is defined as

$$
f_{r}(g)=\left\{\begin{array}{ll}
g \odot P_{2} r, & \text { if } g \in R \\
r, & \text { if } g=a
\end{array}= \begin{cases}g P_{2} r, & \text { if } g \in R \\
r, & \text { if } g=a .\end{cases}\right.
$$


Clearly it follows that $\mu: R \rightarrow P^{*}(H)$, defined by $\mu(r)=f_{r}$, is an inclusive homomorphism, so the general left hypernear-ring $\left(R, \oplus_{P_{1}}, \odot_{P_{2}}\right)$ is W-embeddable in $T^{*}(H)$.

Example 2. Consider the semigroup $(\mathbb{N}, \cdot)$ of natural numbers with the standard multiplication operation and the order " $\leq$ ". Define on it the hyperoperations $+\leq$ and $\cdot \leq$ as follows:

$$
\begin{gathered}
x+\leq y=\{z \mid x \leq z \vee y \leq z\} \\
x \cdot \leq y=\{z \mid x \cdot y \leq z\} .
\end{gathered}
$$

Then the structure $\left(\mathbb{N},+_{\leq}, \leq\right)$is a strongly distributive general hypernear-ring (in fact it is a hyperring). This follows from Theorem 4.3 [19]. Furthermore, for any $a \notin \mathbb{N}$, it can be easily verified that $\left(\mathbb{N},+_{\leq}\right)$is a proper subhypergroup of $\left(\mathbb{N} \cup\{a\},+_{\leq}^{\prime}\right)$, where the hyperoperation $+_{\leq}^{\prime}$ is defined by:

$$
x++_{\leq}^{\prime} y= \begin{cases}x+\leq y, & \text { if } x, y \in \mathbb{N} \\ \mathbb{N} \cup\{a\}, & \text { if } x=a \vee y=a .\end{cases}
$$

In this case, for a fixed $n \in \mathbb{N}$, we can define the multimapping $f_{n}: \mathbb{N} \cup\{a\} \rightarrow P^{*}(\mathbb{N} \cup\{a\})$ as follows:

$$
f_{n}(g)=\left\{\begin{array}{ll}
g \cdot \leq n, & \text { if } g \in \mathbb{N} \\
n, & \text { if } g=a
\end{array}= \begin{cases}\{k \in \mathbb{N} \mid g \cdot n \leq k\}, & \text { if } g \in \mathbb{N} \\
n, & \text { if } g=a\end{cases}\right.
$$

and therefore the mapping $\mu: \mathbb{N} \rightarrow P^{*}(\mathbb{N} \cup\{a\})$ is an inclusive homomorphism. Again this shows that the general hypernear-ring $\left(\mathbb{N},+_{\leq} \cdot \leq\right)$ is $W$-embeddable in $T^{*}(\mathbb{N} \cup\{a\})$.

Example 3. Let $R=\{0,1,2,3\}$. Consider now the semigroup $(R, \cdot)$ defined by Table 1:

Table 1. The Cayley table of the semigroup $(R, \cdot)$

\begin{tabular}{lllll}
\hline$\cdot$ & $\mathbf{0}$ & $\mathbf{1}$ & $\mathbf{2}$ & $\mathbf{3}$ \\
\hline $\mathbf{0}$ & 0 & 0 & 0 & 0 \\
$\mathbf{1}$ & 0 & 1 & 2 & 3 \\
$\mathbf{2}$ & 0 & 1 & 2 & 3 \\
$\mathbf{3}$ & 0 & 1 & 2 & 3 \\
\hline
\end{tabular}

Define on $R$ the hyperoperation $+_{\leq}$as follows: $x+\leq y=\{z \mid x \leq z \vee y \leq z\}$, so its Cayley table is described in Table 2:

Table 2. The Cayley table of the hypergroupoid $(R,+\leq)$

\begin{tabular}{ccccc}
\hline$+\leq$ & $\mathbf{0}$ & $\mathbf{1}$ & $\mathbf{2}$ & $\mathbf{3}$ \\
\hline $\mathbf{0}$ & $\mathrm{R}$ & $\mathrm{R}$ & $\mathrm{R}$ & $\mathrm{R}$ \\
$\mathbf{1}$ & $\mathrm{R}$ & $\{1,2,3\}$ & $\{1,2,3\}$ & $\{1,2,3\}$ \\
$\mathbf{2}$ & $\mathrm{R}$ & $\{1,2,3\}$ & $\{2,3\}$ & $\{2,3\}$ \\
$\mathbf{3}$ & $\mathrm{R}$ & $\{1,2,3\}$ & $\{2,3\}$ & $\{3\}$ \\
\hline
\end{tabular}

Obviously, the relation $\leq$ is reflexive and transitive and, for all $x, y, z \in R$, it holds: $x \leq y \Rightarrow z \cdot x \leq z \cdot y$. Thus, $\left(R,+_{\leq}, \cdot\right)$ is an (additive) hypernear-ring. Let $H=R \cup\{4\}$ and define the hyperoperation $+\leq$ as follows:

$$
x+\leq y=\left\{\begin{array}{cc}
x+\leq y, & \text { if } x, y \in\{0,1,2,3\} \\
H, & \text { if } x=4 \vee y=4
\end{array}\right.
$$


It follows that $(R,+)$ is a proper subhypergroup of $(H,+)$ and for a fixed $r \in R$ it holds $f_{r}(x)=r$, for all $x \in H$. This implies that the mapping $\mu: H \rightarrow P^{*}(H)$, defined by $\mu(r)=f_{r}$ for any $r \in R$, is an inclusive homomorphism.

Now we will construct a left general additive hypernear-ring associated with an arbitrary hypergroup.

Theorem 6. Let $(H,+)$ be a hypergroup and $T(H)=\{f: H \rightarrow H\}$. On the set $T(H)$ define the hyperoperation $\oplus_{T}$ and the operation $\odot_{T}$ as follows:

$$
\begin{gathered}
f \oplus_{T} g=\{h \in T(H) \mid(\forall x \in H) h(x) \in f(x)+g(x)\}, \\
\left(f \odot_{T} g\right)(x)=g(f(x)), \text { for all } x \in H .
\end{gathered}
$$

The obtained structure $\left(T(H), \oplus_{T}, \odot_{T}\right)$ is a (left) general additive hypernear-ring.

Proof. Let $f, g \in T(H)$. We prove that there exists $h \in T(H)$ such that $h(x) \in f(x)+g(x)$ for all $x \in H$. Let $x \in H$. Since $f(x)+g(x) \neq \varnothing$ we can choose $h_{x} \in f(x)+g(x)$ and define $h(x)=h_{x}$. Obviously, $h \in f \oplus_{T} g$. Now we prove that the hyperoperation $\oplus_{T}$ is associative. Let $f, g, h \in T(H)$. Set $L=\left(f \oplus_{T} g\right) \oplus_{T} h=\left\{h^{\prime \prime} \mid(\forall x) h^{\prime \prime}(x) \in h^{\prime}(x)+h(x) \wedge h^{\prime}(x) \in f(x)+g(x)\right\}$ and $D=f \oplus_{T}$ $\left(g \oplus_{T} h\right)=\left\{f^{\prime \prime} \mid(\forall x) f^{\prime \prime}(x) \in f(x)+f^{\prime}(x) \wedge f^{\prime}(x) \in g(x)+h(x)\right\}$. Thus, if $h^{\prime \prime} \in L$, then $h^{\prime \prime}(x) \in$ $(f(x)+g(x))+h(x)=f(x)+(g(x)+h(x))$. Thereby, for any $x \in H$, there exists $a_{x} \in g(x)+h(x)$ such that $h^{\prime \prime}(x) \in f(x)+a_{x}$. Define $f^{\prime}(x)=a_{x}$. Then, $f^{\prime} \in g \oplus_{T} h$ and for all $x \in H$ it holds $h^{\prime \prime}(x) \in f(x)+f^{\prime}(x)$. Therefore, $h^{\prime \prime} \in D$. So, $L \subseteq D$. Similarly, we obtain that $D \subseteq L$. Now, let $f, g \in T(H)$. We prove that the equation $f \in g \oplus_{T} h$ has a solution $h \in T(H)$. Since $(H,+)$ is a hypergroup, it follows that, for any $x \in H$, there exists $b_{x} \in H$ such that $f(x) \in g(x)+b_{x}$. Define $h: H \rightarrow H$ by $h(x)=b_{x}$. Then $h \in T(H)$ and $f \in g \oplus_{T} h$. Similarly, we obtain that the equation $f \in h \oplus_{T} g$ has a solution in $T(H)$. We may conclude that $\left(T(H), \oplus_{T}\right)$ is a hypergroup.

Obviously, $\left(T(H), \odot_{T}\right)$ is a semigroup, because the composition of functions is associative. Now we prove that the hyperoperation $\oplus_{T}$ is left inclusively distributive with respect to the operation $\odot_{T}$. Let $f, g, h \in T(H)$. Set $L=f \odot_{T}\left(g \oplus_{T} h\right)=\left\{f \odot_{T} k \mid k \in g \oplus_{T} h\right\}$ and $D=\left(f \odot_{T} g\right) \oplus_{T}\left(f \odot_{T} h\right)=\left\{h^{\prime} \mid(\forall x \in H) h^{\prime}(x) \in g(f(x))+h(f(x))\right\}$. Let $k \in g \oplus h$. Then, for all $x \in H$, it holds $(f \odot k)(x)=k(f(x)) \subseteq g(f(x))+h(f(x))$. Thus, $f \odot k \in D$, meaning that $L \subseteq D$.

For an arbitrary group $G$, Malone and Heatherly [2] denote by $T_{0}(G)$ the subset of $T(G)$ consisting of the functions which commute multiplicatively with the zero-function, i.e., $T_{0}(G)=\{f: G \rightarrow G \mid$ $f(0)=0\}$. Obviously, $T_{0}(G)$ is a sub-near-ring of $(T(G),+, \cdot)$. The next result extends this property to the case of hyperstructures.

Theorem 7. Let $(H,+)$ be a hypergroup with the identity element 0 (i.e., for all $x \in H$, it holds $x \in$ $x+0 \cap 0+x)$, such that $0+0=\{0\}$. Let $T_{0}(H)=\{f: H \rightarrow H \mid f(0)=0\}$. Then, $T_{0}(H)$ is a subhypernear-ring of the general additive hypernear-ring $\left(T(H), \oplus_{T}, \odot_{T}\right)$.

Proof. Let $f, g \in T_{0}(H)$. If $h \in f \oplus_{T} g$, then $h(0) \in f(0)+g(0)=0+0=\{0\}$, i.e., $h(0)=0$. Thus, $h \in T_{0}(H)$. Let $f, g \in T_{0}(H)$. We prove now that the equation $f \in g \oplus a$ has a solution $a \in T_{0}(H)$. If we set $a(0)=0$ and $a(x)=a_{x}$, where $f(x) \in g(x)+a_{x}$, for $x \neq 0$ and $a_{x} \in H$, then $a \in T_{0}(H)$ and $f \in g+a$. Similarly the equation $f \in a \oplus g$ has a solution $a \in T_{0}(H)$. Thus, $\left(T_{0}(H), \oplus_{T}\right)$ is a subhypergroup of $\left(T(H), \oplus_{T}\right)$. Obviously, if $f, g \in T_{0}(H)$, then it follows that $\left(f \odot_{T} g\right)(0)=g(f(0))=g(0)=0$, i.e., $f \odot_{T} g \in T_{0}(H)$. So, $\left(T_{0}(H), \odot_{T}\right)$ is a subsemihypergroup of $\left(T(H), \odot_{T}\right)$, implying that $T_{0}(H)$ is a subsemihypernear-ring of $T(H)$. 
Theorem 8. Let $(R,+, \cdot)$ be an additive hypernear-ring such that $(R,+)$ is a proper subhypergroup of the hypergroup $(H,+)$, having an identity element 0 satisfying the following properties:

1. $0+0=\{0\}$ and

2. $0 \cdot r=0$, for all $r \in R$.

Then the hypernear-ring $(R,+, \cdot)$ is $W$-embeddable in the additive hypernear-ring $T_{0}(H)$.

Proof. For a fixed $r \in R$, define a map $f: H \rightarrow H$ as follows

$$
f_{r}(g)= \begin{cases}g \cdot r, & \text { if } g \in R \\ r, & \text { if } g \in H \backslash R .\end{cases}
$$

Obviously, $f_{r}(0)=0 \cdot r=0$. So, $f_{r} \in T_{0}(H)$ and, similarly as in the proof of Theorem 5, we obtain that the map $\rho:(R,+, \cdot) \rightarrow\left(T_{0}(H), \oplus_{T}, \odot_{T}\right)$ defined by $\rho(r)=f_{r}$ is an injective inclusion homomorphism.

Example 4. On the set $H=\{0,1,2,3,4,5,6\}$ define an additive hyperoperation and a multiplicative operation having the Cayley tables described in Tables 3 and 4, respectively:

Table 3. The Cayley table of the hypergroupoid $(H,+)$

\begin{tabular}{cccccccc}
\hline+ & $\mathbf{0}$ & $\mathbf{1}$ & $\mathbf{2}$ & $\mathbf{3}$ & $\mathbf{4}$ & $\mathbf{5}$ & $\mathbf{6}$ \\
\hline $\mathbf{0}$ & 0 & 1 & 2 & 3 & 4 & 5 & 6 \\
$\mathbf{1}$ & 1 & 2 & 3 & 4 & 5 & $\{0,6\}$ & 1 \\
$\mathbf{2}$ & 2 & 3 & 4 & 5 & $\{0,6\}$ & 1 & 2 \\
$\mathbf{3}$ & 3 & 4 & 5 & $\{0,6\}$ & 1 & 2 & 3 \\
$\mathbf{4}$ & 4 & 5 & $\{0,6\}$ & 1 & 2 & 3 & 4 \\
$\mathbf{5}$ & 5 & $\{0,6\}$ & 1 & 2 & 3 & 4 & 5 \\
$\mathbf{6}$ & 6 & 1 & 2 & 3 & 4 & 5 & 0 \\
\hline
\end{tabular}

Table 4. The Cayley table of the semigroup $(H, \cdot)$

\begin{tabular}{llllllll}
\hline & $\mathbf{0}$ & $\mathbf{1}$ & $\mathbf{2}$ & $\mathbf{3}$ & $\mathbf{4}$ & $\mathbf{5}$ & $\mathbf{6}$ \\
\hline $\mathbf{0}$ & 0 & 0 & 0 & 0 & 0 & 0 & 0 \\
$\mathbf{1}$ & 0 & 5 & 4 & 3 & 2 & 1 & 0 \\
$\mathbf{2}$ & 0 & 1 & 2 & 3 & 4 & 5 & 0 \\
$\mathbf{3}$ & 0 & 0 & 0 & 0 & 0 & 0 & 0 \\
$\mathbf{4}$ & 0 & 5 & 4 & 3 & 2 & 1 & 0 \\
$\mathbf{5}$ & 0 & 1 & 2 & 3 & 4 & 5 & 0 \\
$\mathbf{6}$ & 0 & 0 & 0 & 0 & 0 & 0 & 0 \\
\hline
\end{tabular}

The structure $(H,+, \cdot)$ is an (additive) hypernear-ring [16].

Let $R=\{0,3,6\}$. Then $(R,+, \cdot)$ is a hypernear-ring (in particular it is a subhypernear-ring of $(H,+, \cdot)$ ). Obviously, $(R,+)$ is a proper subhypergroup of the hypergroup $(H,+)$, which has the identity 0 such that $0+0=\{0\}$ and $0 \cdot r=0$, for all $r \in R$. It follows that, for each $r \in\{0,3,6\}, f_{r}: H \rightarrow H$ is a map such that $f_{0}(g)=0$, for all $g \in H$,

$f_{3}(g)=\left\{\begin{array}{cl}g \cdot 3, & \text { if } g \in\{0,3,6\} \\ 3, & \text { if } g \in\{1,2,4\}\end{array}= \begin{cases}0, & \text { if } g \in\{0,3,6\} \\ 3, & \text { if } g \in\{1,2,4\},\end{cases}\right.$

while

$f_{6}(g)=\left\{\begin{array}{cl}g \cdot 6, & \text { if } g \in\{0,3,6\} \\ 6, & \text { if } g \in\{1,2,4\}\end{array}= \begin{cases}0, & \text { if } g \in\{0,3,6\} \\ 6, & \text { if } g \in\{1,2,4\} .\end{cases}\right.$ 
Clearly, the map $\rho:(R,+, \cdot) \rightarrow\left(T_{0}(H), \oplus_{T}, \odot_{T}\right)$, defined by $\rho(r)=f_{r}$, is an injective inclusion homomorphism, so the hypernear-ring $R$ is $W$-embeddable in $T_{0}(H)$.

Remark 3. If $(G,+)$ is a group, then, for any $f, g \in T(G)=\{f: G \rightarrow G\}$, it holds $f \oplus_{T} g=f+g$ and $f \odot_{T} g=f \cdot g$, meaning that the transformation near-ring $(T(G),+, \cdot)$ of a group $G$ is in fact the structure $\left(T(G), \oplus_{T}, \odot_{T}\right)$. Furthermore, if $(R,+, \cdot)$ is a zero-symmetric near-ring, i.e., a near-ring in which any element $x$ satisfies the relation $x \cdot 0=0 \cdot x=0$, then the map $\rho$ constructed in the proof of Theorem 8 is the injective homomorphism $\rho: R \rightarrow T_{0}(G)$. Thus, according with Theorem 8, it follows that the zero-symmetric near-ring $(R,+, \cdot)$ is $W$-embeddable in the near-ring $T_{0}(G)$, where $(G,+)$ is any group containing $(R,+)$ as a proper subgroup.

Remark 4. If $(G,+)$ is a group, then the following inclusions hold: $T_{0}(G) \subseteq T(G) \subseteq T^{*}(G)$, where both $T(G)$ and $T_{0}(G)$ are sub-(hyper)near-rings of the hypernear-ring $T^{*}(G)$. Considering now $(H,+)$ a hypergroup, the same inclusions exist: $T_{0}(H) \subseteq T(H) \subseteq T^{*}(H)$, but generally $T(H)$ and $T_{0}(H)$ are not subhypernear-rings of $T^{*}(H)$.

Proposition 1. Let $(H,+)$ be a hypergroup with the identity element 0 (i.e., for all $x \in H$ it holds $x \in$ $x+0 \cap 0+x)$ such that $0+0=\{0\}$. Let $T_{0}^{*}(H)=\left\{f: H \rightarrow P^{*}(H) \mid f(0)=0\right\}$. Then, $T_{0}^{*}(H)$ is a subhypernear-ring of the general hypernear-ring $\left(T^{*}(H), \oplus, \odot\right)$.

Proof. Let $f, g \in T_{0}^{*}(H)$. If $h \in f \oplus g$, then it holds $h(0) \subseteq f(0)+g(0)=0+0=\{0\}$. Since $h(0) \neq \varnothing$, it follows that $h(0)=\{0\}$. Thus, $h \in T_{0}^{*}(H)$. Let $f, g \in T_{0}^{*}(H)$. We prove that the equation $f \in g \oplus a$ has a solution $a \in T_{0}^{*}(H)$. If we set $a(0)=0$ and $a(x)=H$, for all $x \neq 0$, then $a \in T_{0}^{*}(H)$ and, for all $x \neq 0$, it holds $g(x)+a(x)=H \supseteq f(x)$ and $g(0)+a(0)=\{0\}=f(0)$, meaning that $f \in g \oplus a$. Similarly, the equation $f \in a \oplus g$ has a solution in $T_{0}^{*}(H)$. So, $\left(T_{0}^{*}(H)\right)$ is a subhypergroup of $\left(T^{*}(H), \oplus\right)$. Obviously, if $h \in f \odot g$, then $h(0) \subseteq g(f(0))=\{0\}$. So, $h \in T_{0}^{*}(H)$. Thus $T_{0}^{*}(H)$ is a subsemihypergroup of $\left(T^{*}(H), \odot\right)$. Therefore, $T_{0}^{*}(H)$ is a subhypernear-ring of $\left(T^{*}(H), \oplus, \odot\right)$.

\section{Conclusions}

Distributivity property plays a fundamental role in the ring-like structures, i.e., algebraic structures endowed with two operations, usually denoted by addition and multiplication, where the multiplication distributes over the addition. If this happens only from one-hand side, then we talk about near-rings. Similarly, in the framework of algebraic hypercompositional structures, a general hypernear-ring has the additive part an arbitrary hypergroup, the multiplicative part is a semihypergroup, and the multiplication hyperoperation inclusively distributes over the hyperaddition from the left or right-hand side, i.e., for three arbitrary elements $x, y, z$, there is $x \cdot(y+z) \subseteq x \cdot y+x \cdot z$ for the left-hand side, and respectively, $(y+z) \cdot x \subseteq y \cdot x+z \cdot x$ for the right-hand side. If the inclusion is substituted by equality, then the general hypernear-ring is called strongly distributive. We also recall here that there exist also hyperrings having the additive part a group, while the multiplicative one is a semihypergroup, being called multiplicative hyperrings [20].

The set of all transformations of a group $G$, i.e., $T(G)=\{g: G \rightarrow G\}$, can be endowed with a near-ring structure, while similarly, on the set of all multitransformations of a hypergroup $H$, i.e., $T^{*}(H)=\left\{h: H \rightarrow \mathcal{P}^{*}(H)\right\}$, can be defined a general hypernear-ring structure, called the multitransformations general hypernear-ring associated with the hypergroup $H$. We have shown that for every general hypernear-ring $R$ there exists a hypergroup $H$ such that $R$ is weakly embeddable in the associated multitransformations general hypernear-ring $T^{*}(H)$ (see Theorem 5). Moreover, considering the set $T(H)=\{f: H \rightarrow H\}$ of all transformations of a hypergroup $H$, we have defined on it a hyperaddition and a multiplication such that $T(H)$ becomes a general additive hypernear-ring. We have determined conditions under which the set $T_{0}(H)$, formed with the transformations of $H$ that multiplicatively commute with the zero function on $H$, is a subhypernear-ring of $T(H)$. Besides, 
an additive hypernear-ring satisfying certain conditions can be weakly embedded in the additive hypernear-ring $T_{0}(H)$ (see Theorem 8).

In our future work, we intend to introduce and study properties of $\Delta$-endomorphisms and $\Delta$-multiendomorphisms of hypernear-rings as generalizations of similar notions on near-rings.

Author Contributions: The authors contributed equally to this paper.

Funding: The third author acknowledges the financial support from the Slovenian Research Agency (research core funding No. P1-0285).

Conflicts of Interest: The authors declare no conflict of interest.

\section{References}

1. Berman, G.; Silverman, R.J. Near-rings. Am. Math. Mon. 1959, 66, 23-34. [CrossRef]

2. Malone, J.J.; Heatherly, H.E., Jr. Some Near-Ring Embeddings. Quart. J. Math. Oxf. Ser. 1969, 20, 81-85. [CrossRef]

3. Dašić, V. Hypernear-rings. In Algebraic Hyperstructures and Applications (Xanthi, 1990); World Scientific Publishing: Teaneck, NJ, USA, 1991; pp. 75-85.

4. Bonansinga, P. Sugli ipergruppi quasicanonici. Atti Soc. Peloritana Sci. Fis. Mat. Natur 1981, 27, 9-17.

5. Massouros, C.G. Quasicanonical hypergroups. In Algebraic Hyperstructures and Applications (Xanthi, 1990); World Scientific Publishing: Teaneck, NJ, USA, 1991; pp. 129-136.

6. Comer, S.D. Polygroups derived from cogroups. J. Algebra 1984, 89, 387-405. [CrossRef]

7. Davvaz, B. Polygroup Theory and Related Systems; World Scientific Publishing, Co. Pte. Ltd.; Hackensack, NJ, USA, 2013.

8. Jančić-Rašović, S.; Cristea, I. A note on near-rings and hypernear-rings with a defect of distributivity. AIP Conf. Proc. 1978, 1978, 34007.

9. Ameri, R.; Amiri-Bideshki, M.; Hoskova-Mayerova, S.; Saeid, A.B. Distributive and Dual Distributive Elements in Hyperlattices. Ann. Univ. Ovidius Constanta Ser. Mat. 2017, 25, 25-36. [CrossRef]

10. Jančíć-Rašović, S.; Cristea, I. Division hypernear-rings. Ann. Univ. Ovidius Constanta Ser. Mat. 2018, 26, 109-126. [CrossRef]

11. Gontineac, M. On Hypernear-ring and H-hypergroups. In Algebraic Hyperstructures and Applications (Lasi, 1993); Hadronic Press: Palm Harbor, FL, USA, 1994; pp. 171-179.

12. Dašić, V. A defect of distributivity of the near-rings. Math. Balk. 1978, 8, 63-75.

13. Clay, J. Nearrings: Geneses and Application; Oxford University Press: Oxford, UK, 1992.

14. Meldrum, J. Near-Rings and Their Links with Groups; Pitman: London, UK, 1985.

15. Pilz, G. Near-Rings: The theory and Its Applications; North-Holland Publication Co.: New York, NY, USA, 1983.

16. Jančić-Rašović, S.; Cristea, I. Hypernear-rings with a defect of distributivity. Filomat 2018, 32, 1133-1149. [CrossRef]

17. Vougiouklis, T. Hyperstructures and Their Representations; Hadronic Press: Palm Harbor, FL, USA, 1994.

18. Jančić-Rašović, S. On a class of $P_{1}-P_{2}$ hyperrings and hypernear-rings. Set-Val. Math. Appl. 2008, 1, 25-37.

19. Jančić-Rašović, S.; Dasic, V. Some new classes of $(m, n)$-hyperrings. Filomat 2012, 26, 585-596. [CrossRef]

20. Ameri, R.; Kordi, A.; Hoškova-Mayerova, S. Multiplicative hyperring of fractions and coprime hyperideals. Ann. Univ. Ovidius Constanta Ser. Mat. 2017, 25, 5-23. [CrossRef] 